\title{
The Error-Related Negativity (ERN) in Anxiety and Obsessive-Compulsive Disorder (OCD): A Call for Further Investigation of Task Parameters in the Flanker Task
}

\author{
Lilianne M. Gloe ${ }^{\star \dagger}$ and Courtney C. Louis ${ }^{\dagger}$ \\ Department of Psychology, Michigan State University, East Lansing, MI, United States
}

Keywords: ERN (error-related negativity), obsessive-compulsive disorder, anxiety, Flanker task, task parameters

\section{OPEN ACCESS}

Edited by:

Nico Bunzeck,

University of Lübeck, Germany

Reviewed by:

Nora Alicia Herweg,

University of Pennsylvania,

United States

Ana Isabel Rosa-Alcázar,

University of Murcia, Spain

${ }^{*}$ Correspondence:

Lilianne M. Gloe

rothsch7@msu.edu

tThese authors have contributed equally to this work and share first

authorship

Specialty section:

This article was submitted to

Cognitive Neuroscience,

a section of the journal

Frontiers in Human Neuroscience

Received: 17 September 2021 Accepted: 23 November 2021 Published: 09 December 2021

Citation:

Gloe LM and Louis CC (2021) The Error-Related Negativity (ERN) in

Anxiety and Obsessive-Compulsive

Disorder (OCD): A Call for Further Investigation of Task Parameters in the

Flanker Task.

Front. Hum. Neurosci. 15:779083.

doi: 10.3389/fnhum.2021.779083

\section{INTRODUCTION}

The study of biomarkers and endophenotypes has proliferated in human clinical neuroscience in recent decades, with the hope that specific physiological signals may hold unique information for assessing psychopathology. Additionally, many researchers have sought to understand functional relationships between psychopathology and physiological phenomena to illuminate potential mechanisms and impacts of psychopathology. Both endophenotype and functional approaches have been used to interpret how anxiety ${ }^{1}$ and obsessive-compulsive disorder (OCD) relate to the error-related negativity (ERN), a negative-going signal that peaks within $100 \mathrm{~ms}$ after error commissions at frontocentral recording sites measured via electroencephalogram (EEG; Gehring et al., 2012; for reviews: Moser et al., 2013; Riesel, 2019). The functional significance of the ERN continues to be debated (for prominent theories, see Holroyd and Coles, 2002; Yeung et al., 2004; Holroyd et al., 2005; Gehring et al., 2012). However, it is generally accepted that the ERN is implicated in the error-monitoring process (Gehring et al., 2012). The ERN is likely generated by the dorsal region of the anterior cingulate cortex (ACC), an area highly responsive to conflict (Carter et al., 1998; Taylor et al., 2007), and influenced by activity in motor areas, such as the pre-supplementary motor area (pre-SMA; Hochman et al., 2009; Iannaccone et al., 2015). Meta-analytic evidence has demonstrated that the ERN is enhanced for those with high anxiety and OCD (for review: Moser et al., 2013, 2016; Riesel, 2019), and has highlighted the ERN as a promising physiological signal to understand these disorders.

Importantly, however, heterogeneity in the magnitude of the anxiety/OCD-ERN association has been identified (for review: Saunders and Inzlicht, 2020). Some of this variability in adults has been explained by moderators, such as sex and anxiety symptom dimensions (for review: Moser et al., 2016). Despite this, unexplained heterogeneity in effect sizes remains between studies. Further, a recent meta-analysis found that the magnitude of these effects is influenced by publication bias (Saunders and Inzlicht, 2020), suggesting that findings may be even more heterogeneous than indicated by the published literature. Therefore, additional moderators are likely present, influencing effect sizes and the utility of the ERN as an individual difference metric (Clayson et al., 2021).

One class of moderators that requires further elucidation are task parameters of the Flanker task, a commonly used task to elicit the ERN in the context of anxiety and OCD. The Flanker task is a

${ }^{1}$ In the text, we use the term "worry" to refer to a specific cognitive dimension of anxiety, and the term "anxiety" to refer to both worry and somatic dimensions of anxiety (Heller et al., 1997; Nitschke et al., 2001). 
speeded two-choice response task (Eriksen and Eriksen, 1974). In the traditional version of the task, participants are asked to respond to a center letter (i.e., the target) in the presence of several flanking distractor letters that can either be congruent (e.g., $\mathrm{HHHHH}$ ) or incongruent (e.g., HHSHH) with the target. Notably, many studies have used a version of the Flanker task with modified arrow stimuli (e.g., $<<<<<$ and $<<><<$ ). Although the Flanker task is a commonly used task in studies of the anxiety/OCD-ERN association, there is great heterogeneity in the task parameters used across studies. We consider how common variations in Flanker task parameters may influence the magnitude of the anxiety/OCD-ERN association.

\section{TASK MANIPULATIONS AND ANXIETY/OCD-ERN ASSOCIATION}

\section{The Role of Feedback in the Anxiety/OCD-ERN Association}

One such manipulation of the Flanker task that varies between studies of anxiety/OCD and the ERN is the frequency and content of performance feedback provided to participants during the task. Many studies of anxiety/OCD and the ERN use a version of the task that provides indicators of performance at block breaks (e.g., Riesel et al., 2011; Klawohn et al., 2014; Weinberg et al., 2015). If participants fall below an accuracy threshold (e.g., $<80 \%$ accuracy) they are told to respond more accurately following a block of trials. Alternatively, if performance falls above a particular accuracy threshold (e.g., >90\%), participants are told to respond faster following a block of trials. Some versions of the task provide performance-based feedback after each trial (e.g., Cavanagh and Allen, 2008; Endrass et al., 2010; Xiao et al., 2011). Additionally, researchers have also used a paradigm in which no performance feedback is provided (e.g., Moran et al., 2012; Schroder et al., 2017; Riesel et al., 2019a,b).

In non-clinical samples, task instructions that emphasize accuracy over speed result in an enhanced ERN (Gehring et al., 1993; Arbel and Donchin, 2009, but see also Coleman et al., 2018). In contrast, when task instructions emphasize speed over accuracy, the ERN amplitude is diminished (Gehring et al., 1993). In studies of anxiety and OCD, performance-based feedback may moderate the anxiety/OCDERN association by altering relative emphasis on speed and accuracy. Riesel et al. (2019a,b) demonstrated that when accuracy was emphasized through task instruction and trialto-trial feedback, healthy controls experienced an increase in the ERN, such that it was not significantly different from participants diagnosed with OCD. On the other hand, an enlarged ERN in OCD was still found in comparison to healthy controls when speed was emphasized through task instruction and trial-to-trial feedback (Riesel et al., 2019a,b). These findings provide compelling evidence that the task's relative emphasis on accuracy or speed may alter comparisons between those with and without OCD. Riesel and colleagues argue that individuals with OCD may experience difficulty adapting to different contexts and present with a more fixed response style. Additionally, Olvet and Hajcak (2009) identified that the ERN-anxiety association was not significant when trial-to-trial accuracy-based feedback was provided; however, the association did emerge when no trial-to-trial feedback was given. Olvet and Hajcak (2009) argue that feedback may reduce the load of error monitoring by providing anxious individuals with feedback on their performance. Therefore, existing evidence suggests that trial-to-trial feedback may influence the magnitude of the ERN in both OCD and anxiety, such that emphasizing accuracy results in a dampened association.

While no studies have examined the effect of block-to-block feedback on the anxiety/OCD-ERN association specifically, we hypothesize that providing different levels of accuracy emphasis to individuals results in a dampening of the association in tasks that use block-to-block feedback in comparison to those that use no feedback. For example, if Participant A makes more errors across blocks than Participant B, Participant A will receive more accuracy-based feedback. If Participant A also has relatively few blocks where high accuracy is achieved and, thus, receives little feedback about the speed of their performance, accuracy would be over-emphasized in feedback relative to speed. In contrast, Participant B who makes either a few or a moderate number of errors across blocks would either (1) receive more speedrelated feedback because of high block accuracy or (2) minimal feedback about accuracy or speed because of average accuracy on block performance. Thus, the sample could be relatively heterogeneous for the relative proportions of speed and accuracy feedback, creating systematic, unaccounted for variability in the anxiety/OCD-ERN association.

\section{Other Task Factors to Consider in the Anxiety/OCD-ERN Association}

In addition to performance-related feedback, future work should examine the role of task stimuli and mode of response. In a preliminary study, Lin et al. (2015) examined whether the association between worry and the ERN differed between tasks that used vertically and horizontally presented arrows, given that both presentations are regularly employed in studies of anxiety and the ERN. They found that worry was only associated with the ERN when arrow stimuli were presented horizontally (Lin et al., 2015). Lin et al. (2015) interprets these findings in the context of the Compensatory Error Monitoring Hypothesis (CEMH), which states that an enhanced ERN reflects compensatory recruitment of cognitive resources due to the taxing effect of worry (Moser et al., 2013). Specifically, Lin et al. (2015) theorize that worry utilizes verbal resources that are also being drawn upon during the processing of horizontal representations (i.e., reading). It may be that the association between worry/GAD and the ERN is more likely to emerge with horizontal stimuli because they are more cognitively demanding for worriers than vertical stimuli (Lin et al., 2015). Notably, no studies have examined whether effect sizes are influenced by the use of arrows as opposed to letters for stimuli, despite both versions of the stimuli being employed regularly in this literature. In addition, no studies have investigated whether the stimuli orientation (i.e., vertical vs. 
horizontal) influence the magnitude of the ERN in OCD or other types of anxiety besides worry.

Additionally, Flanker task versions differ in response mappings, such that some studies require participants to respond with index fingers on each hand, thumbs on each hand or the index and middle fingers on the same hand. Lin et al. (2015) also found that when using the left and right index finger to respond, worry was only related to the ERN on trials where errors were made with the right index finger during horizontal stimuli presentations, irrespective of participant handedness. In line with CEMH, the authors discuss that the worry-ERN association is larger with right-handed errors due to enhanced conflict driven by left-hemisphere verbal processing during horizontal stimulus presentation (Lin et al., 2015). That is, because verbal processing is left-lateralized and right-handed responses are controlled by the left-hemisphere, this increased conflict during right-handed errors results in an enhanced ERN magnitude. Indeed, others have suggested that response mapping factors, such as hand of error and even finger of error, influence the amplitude of the ERN itself (Hochman et al., 2014). Yet, these factors have yet to be considered in the empirical literature examining the anxiety/OCD-ERN association.

Finally, many versions of the letter flanker task include "switch blocks," in which the stimulus-response mapping from the previous block is reversed. For example, the first block of a task may instruct participants to use their index finger to respond when an " $\mathrm{M}$ " is the target (i.e., center letter), while on the second block (i.e., the switch block) participants may be asked to use their middle finger to respond when an " $\mathrm{M}$ " is the target. In a non-anxious sample, one study found that the ERN is enhanced during switch blocks in comparison to non-switch blocks, speculated to be reflective of enhanced response conflict (Schroder et al., 2012). That is, switch blocks may function as a task-switching component of the flanker, during which the inhibition of previously learned stimulusresponse mappings is required (Schroder et al., 2012). Because the ERN magnitude is larger on switch blocks, there could be variability in the association between the anxiety/OCD and the ERN on switch blocks, specifically, that has yet to be uncovered. Therefore, it will be important for studies to consider switch blocks as a within-subject moderator of the anxiety/OCD-ERN association.

\section{CONCLUSION}

An impressive body of work has been generated over recent decades examining the association between anxiety/OCD and

\section{REFERENCES}

Arbel, Y., and Donchin, E. (2009). Parsing the componential structure of post-error ERPs: a principal component analysis of ERPs following errors. Psychophysiology 46, 1179-1189. doi: 10.1111/j.1469-8986.2009.00857.x

Carter, C. S., Braver, T. S., Barch, D. M., Botvinick, M. M., Noll, D., and Cohen, J. D. (1998). Anterior cingulate cortex, error detection, and the online monitoring of performance. Science 280, 747-749. doi: 10.1126/science.280.5364.747 the ERN. We argue that task parameters, namely feedback, task stimuli, mode of response, response mappings and switch blocks, will be a useful avenue to explore to ultimately enhance clinical theories of the anxiety/OCD-ERN association. We expect that task feedback that emphasizes accuracy would reduce the association between anxiety/OCD and the ERN. Further, we expect that worry, specifically, will have a stronger association with the ERN when responses are made with one's right hand and when stimuli are presented horizontally. Given the lack of literature, it is difficult to predict if other anxiety dimensions (i.e., somatic anxiety) and OCD will function similarly to worry. Finally, we advocate for greater investigation of switch blocks given previous findings indicating that blocks with greater conflict result in a larger ERN in healthy controls. From an endophenotype/biomarker perspective, efforts to use the Flanker task as a clinical assessment tool should use Flanker task versions that maximize the difference between those with and without anxiety or OCD. Additionally, across endophenotype/biomarker and functional perspectives, considering task manipulations may provide further insights into the mechanisms underlying anxiety/OCD-ERN association. By developing this knowledge, the depth and potential applications of this work will continue to burgeon to the benefit of clinical populations.

\section{AUTHOR CONTRIBUTIONS}

LG and CL each wrote sections of the manuscript, contributed to manuscript revision, and approved the submitted version.

\section{FUNDING}

LG was supported by the National Science Foundation Graduate Research Fellowship Program (NSF GRFP, United States, grant number: DGE-1848739) while working on this manuscript. CL was funded by the National Institute of Health Research Supplement to Promote Diversity in HealthRelated Research and the Ruth L. Kirschstein National Research Service Award Individual Predoctoral Fellowship to Promote Diversity in Health-Related Research (NRSA F31 - Diversity, United States, grant number: F31MH125604) while working on this manuscript.

\section{ACKNOWLEDGMENTS}

We would like to thank Drs. Katherine N. Thakkar and Jason S. Moser for their valuable comments on early drafts of this manuscript. 
Coleman, J. R., Watson, J. M., and Strayer, D. L. (2018). Working memory capacity and task goals modulate error-related ERPs. Psychophysiology 55:e12805. doi: $10.1111 /$ psyp. 12805

Endrass, T., Schuermann, B., Kaufmann, C., Spielberg, R., Kniesche, R., and Kathmann, N. (2010). Performance monitoring and error significance in patients with obsessive-compulsive disorder. Biol. Psychol. 84, 257-263. doi: 10.1016/j.biopsycho.2010.02.002

Eriksen, B. A., and Eriksen, C. W. (1974). Effects of noise letters upon the identification of a target letter in a nonsearch task. Percept. Psychophys. 16, 143-149. doi: 10.3758/BF03203267

Gehring, W. J., Goss, B., Coles, M. G., Meyer, D. E., and Donchin, E. (1993). A neural system for error detection and compensation. Psycho sci. 4:385-390.

Gehring, W. J., Liu, Y., Orr, J. M., and Carp, J. (2012). "The error-related negativity (ERN/Ne)," in Oxford Handbook Event-Related Potential Component, eds E. S. Kappenman and S. J. Luck (Oxford: Oxford University Press), 231-291. doi: 10.1093/oxfordhb/9780195374148.013.0120

Heller, W., Nitschke, J. B., Etienne, M. A., and Miller, G. A. (1997). Patterns of regional brain activity differentiate types of anxiety. J. Abnorm. Psychol. 106:376. doi: 10.1037/0021-843X.106.3.376

Hochman, E. Y., Eviatar, Z., Breznitz, Z., Nevat, M., and Shaul, S. (2009). Source localization of error negativity: additional source for corrected errors. NeuroReport. 20:1144-1148.

Hochman, E. Y., Orr, J. M., and Gehring, W. J. (2014). Toward a more sophisticated response representation in theories of medial frontal performance monitoring: the effects of motor similarity and motor asymmetries. Cereb. Cortex 24, 414-425. doi: 10.1093/cercor/bhs323

Holroyd, C. B., and Coles, M. G. (2002). The neural basis of human error processing: reinforcement learning, dopamine, and the error-related negativity. Psychol. Rev. 109:679. doi: 10.1037/0033-295X.109.4.679

Holroyd, C. B., Yeung, N., Coles, M. G. H., and Cohen, J. D. (2005). A Mechanism for Error Detection in Speeded Response Time Tasks. J. Exp. Psychol. Gen. 134, 163-191. doi: 10.1037/0096-3445.134.2.163

Iannaccone, R., Hauser, T. U., Staempfli, P., Walitza, S., Brandeis, D., and Brem, S. (2015). Conflict monitoring and error processing: new insights from simultaneous EEG-fMRI. Neuroimage. 105:395-407.

Klawohn, J., Riesel, A., Grützmann, R., Kathmann, N., and Endrass, T. (2014). Performance monitoring in obsessive-compulsive disorder: a temporo-spatial principal component analysis. Cogn. Affect. Behav. Neurosci. 14, 983-995. doi: 10.3758/s13415-014-0248-0

Lin, Y., Moran, T. P., Schroder, H. S., and Moser, J. S. (2015). The role of hand of error and stimulus orientation in the relationship between worry and errorrelated brain activity: Implications for theory and practice. Psychophysiology 52, 1281-1292. doi: 10.1111/psyp.12470

Moran, T. P., Taylor, D., and Moser, J. S. (2012). Sex moderates the relationship between worry and performance monitoring brain activity in undergraduates. Int. J. Psychophysiol. 85, 188-194. doi: 10.1016/j.ijpsycho.2012.05.005

Moser, J. S., Moran, T. P., Kneip, C., Schroder, H. S., and Larson, M. J. (2016). Sex moderates the association between symptoms of anxiety, but not obsessive compulsive disorder, and error-monitoring brain activity: a meta-analytic review. Psychophysiology 53, 21-29. doi: 10.1111/psyp.12509

Moser, J. S., Moran, T. P., Schroder, H. S., Donnellan, M. B., and Yeung, N. (2013). On the relationship between anxiety and error monitoring: a meta-analysis and conceptual framework. Front. Hum. Neurosci. 7:466. doi: 10.3389/fnhum.2013.00466

Nitschke, J. B., Heller, W., Imig, J. C., McDonald, R. P., and Miller, G. A. (2001). Distinguishing dimensions of anxiety and depression. Cogn. Ther. Res. 25, 1-22. doi: 10.1023/A:1026485530405

Olvet, D. M., and Hajcak, G. (2009). The effect of trial-to-trial feedback on the error-related negativity and its relationship with anxiety. Cogn. Affect. Behav. Neurosci. 9, 427-433. doi: 10.3758/CABN.9.4.427
Riesel, A. (2019). The erring brain: Error-related negativity as an endophenotype for OCD-A review and meta-analysis. Psychophysiology 56:e13348. doi: $10.1111 /$ psyp. 13348

Riesel, A., Endrass, T., Kaufmann, C., and Kathmann, N. (2011). Overactive errorrelated brain activity as a candidate endophenotype for obsessive-compulsive disorder: evidence from unaffected first-degree relatives. Am. J. Psychiatry 168, 317-324. doi: 10.1176/appi.ajp.2010.10030416

Riesel, A., Kathmann, N., and Klawohn, J. (2019a). Flexibility of error-monitoring in obsessive-compulsive disorder under speed and accuracy instructions. $J$. Abnorm. Psychol. 128:671. doi: 10.1037/abn0000463

Riesel, A., Klawohn, J., Grützmann, R., Kaufmann, C., Heinzel, S., Bey, K., et al. (2019b). Error-related brain activity as a transdiagnostic endophenotype for obsessive-compulsive disorder, anxiety and substance use disorder. Psychol. Med. 49, 1207-1217. doi: 10.1017/S00332917190 00199

Saunders, B., and Inzlicht, M. (2020). Assessing and adjusting for publication bias in the relationship between anxiety and the error-related negativity. Int. J. Psychophysiol. 155, 87-98. doi: 10.1016/j.ijpsycho.2020. 05.008

Schroder, H. S., Glazer, J. E., Bennett, K. P., Moran, T. P., and Moser, J. S. (2017). Suppression of error-preceding brain activity explains exaggerated error monitoring in females with worry. Biol. Psychol. 122, 33-41. doi: 10.1016/j.biopsycho.2016.03.013

Schroder, H. S., Moran, T. P., Moser, J. S., and Altmann, E. M. (2012). When the rules are reversed: action-monitoring consequences of reversing stimulus-response mappings. Cogn. Affect. Behav. Neurosci. 12, 629-643. doi: 10.3758/s13415-012-0105-y

Taylor, S. F., Stern, E. R., and Gehring, W. J. (2007). Neural systems for error monitoring: recent findings and theoretical perspectives. Neuroscientist 13, 160-172. doi: 10.1177/1073858406298184

Weinberg, A., Kotov, R., and Proudfit, G. H. (2015). Neural indicators of error processing in generalized anxiety disorder, obsessive-compulsive disorder, and major depressive disorder. J. Abnorm. Psychol. 124:172. doi: 10.1037/abn0000019

Xiao, Z., Wang, J., Zhang, M., Li, H., Tang, Y., Wang, Y., et al. (2011). Error-related negativity abnormalities in generalized anxiety disorder and obsessive-compulsive disorder. Prog. Neuropsychopharmacol. Biol. Psychiatry 35, 265-272. doi: 10.1016/j.pnpbp.2010. 11.022

Yeung, N., Botvinick, M. M., and Cohen, J. D. (2004). The neural basis of error detection: conflict monitoring and the error-related negativity. Psychol. Rev. 111:931. doi: 10.1037/0033-295X.111.4.931

Conflict of Interest: The authors declare that the research was conducted in the absence of any commercial or financial relationships that could be construed as a potential conflict of interest.

Publisher's Note: All claims expressed in this article are solely those of the authors and do not necessarily represent those of their affiliated organizations, or those of the publisher, the editors and the reviewers. Any product that may be evaluated in this article, or claim that may be made by its manufacturer, is not guaranteed or endorsed by the publisher.

Copyright (C) 2021 Gloe and Louis. This is an open-access article distributed under the terms of the Creative Commons Attribution License (CC BY). The use, distribution or reproduction in other forums is permitted, provided the original author(s) and the copyright owner(s) are credited and that the original publication in this journal is cited, in accordance with accepted academic practice. No use, distribution or reproduction is permitted which does not comply with these terms. 